06.3

\title{
Медленные электромагнитные волны в левой среде на основе магнитоактивного плазменного метаматериала
}

\author{
(c) А.В. Богомолова, С.В. Гришин Ф, Ю.П. Шараевский \\ Саратовский национальный исследовательский государственный университет им. Н.Г. Чернышевского, Саратов, Россия \\ ฯ E-mail: sergrsh@yandex.ru
}

Поступило в Редакцию 14 июля 2020 г.

В окончательной редакции 16 сентября 2020 г.

Принято к публикации 16 сентября 2020 г.

\begin{abstract}
Приводятся результаты теоретического исследования спектра медленных электромагнитных волн (ЭМВ), существующих в „левой“ среде на основе магнитоактивного плазменного метаматериала. Исследуемый метаматериал представляет собой полосковый волновод, полностью заполненный продольно замагниченной „холодной“ электронной плазмой, содержащей периодическую структуру из идеально проводящих кольцевых разомкнутых резонаторов. Показано, что в левой среде на основе анизотропной плазмы существуют либо одна (обыкновенная), либо две (обыкновенная и необыкновенная) медленные ЭМВ в зависимости от выбора значения собственной частоты кольцевых разомкнутых резонаторов и магнитной индукции. Установлено, что необыкновенная ЭМВ обладает отрицательной дисперсией, а дисперсионная характеристика обыкновенной ЭМВ содержит участки как с отрицательной, так и с положительной дисперсией.
\end{abstract}

Ключевые слова: метаматериалы, левая среда, магнитоактивная плазма.

DOI: 10.21883/PJTF.2020.24.50426.18464

В настоящее время одним из бурно развивающихся направлений в области материаловедения и нанотехнологий является исследование метаматериалов, представляющих собой композитные структуры со свойствами, отличными от свойств природных материалов [1-3]. Среди метаматериалов наиболее привлекательными объектами являются „левые“ среды, диэлектрическая и магнитная проницаемости которых являются одновременно отрицательными величинами [4]. Интерес к левым средам связан в первую очередь с возможностью реализации отрицательного показателя преломления и с созданием идеальных линз для получения изображений с субволновым разрешением ниже дифракционного предела Аббе. Кроме того, в левой среде распространяется обратная волна, у которой фазовая и групповая скорости направлены в противоположные стороны.

В первых экспериментальных работах для получения левой среды использовались периодические структуры, состоящие из металлических проволок и кольцевых разомкнутых резонаторов (КРР), период следования которых был намного меньше длины электромагнитной волны (ЭМВ) [5,6]. Периодическая система из тонких проводящих проволок давала возможность создать среду плазмонного типа с отрицательной эффективной диэлектрической проницаемостью на частотах ниже плазменной частоты, в то время как периодическая система из КРР использовалась для получения отрицательных значений у эффективной магнитной проницаемости на частотах, находящихся выше собственной частоты КРР. В работе [7] было предложено совместить естественные магнитные свойства ферромагнетика с искусственно созданными электрическими свойствами периодической структуры из идеально проводящих проволок для со- здания левой среды. Было показано, что в левой среде на основе такой гиротропной матрицы, содержащей включения из металлических субволновых элементов, существует одна необыкновенная медленная обратная объемная ЭМВ. Данный подход к созданию левых сред на основе гиротропных матриц можно распространить и на естественные электрические среды, например, в виде магнитоактивной (анизотропной) плазмы [8]. В отличие от левых сред, созданных на основе изотропной плазмы [5,6], левые среды, созданные на основе анизотропной плазмы, должны поддерживать распространение не только обыкновенной, но и необыкновенной обратной ЭМВ. Помимо этого в левой среде на основе замагниченной плазмы открывается возможность управления спектром указанных волн не только за счет изменения собственной частоты КРР путем подбора соответствующих размеров резонаторов, но и за счет изменения величины магнитной индукции.

В настоящей работе на основе простой электродинамической модели полоскового волновода, полностью заполненного продольно замагниченной электронной плазмой, содержащей периодическую структуру КРР, показана возможность создания левой среды, в которой управление спектром обратных ЭМВ осуществляется за счет одновременного изменения собственной частоты КРР и магнитной индукции.

На рис. 1,a приведено схематическое изображение анализируемого плазменного метаматериала. Он представляет собой продольно замагниченный слой плазмы (направление вектора магнитной индукции $\mathbf{B}_{0}$ совпадает с осью $0 Z$ и направлением волнового вектора $\mathbf{k}$ ), внутри которого находится периодическая структура КРР. Для описания магнитных свойств такой периодической 

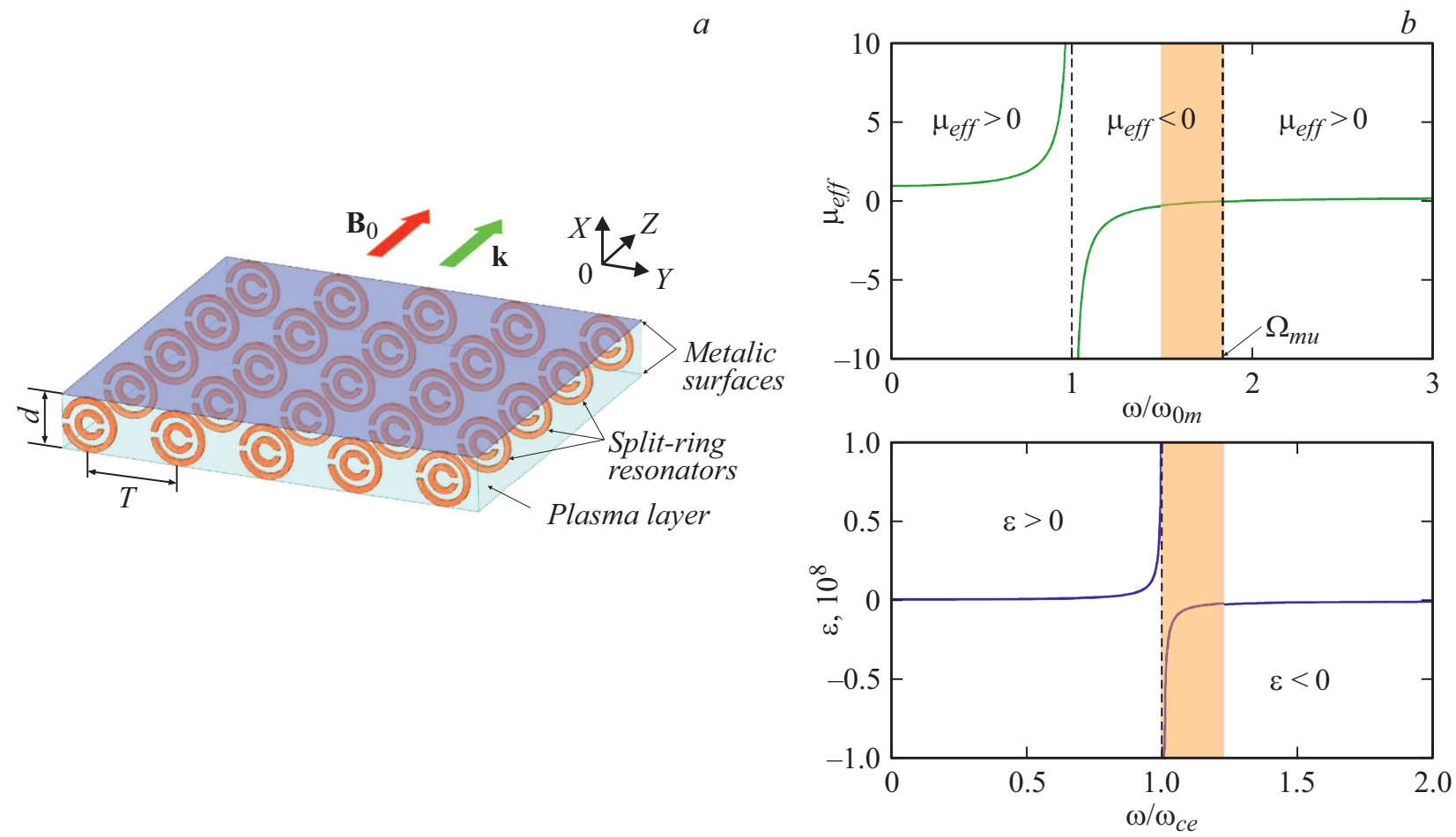

Рис. 1. $a$ - схематическое изображение магнитоактивного плазменного метаматериала, $b$ - зависимости эффективной магнитной проницаемости среды $\mu_{e f f}$ (верхняя панель) и диагональной компоненты $\varepsilon$ тензора высокочастотной диэлектрической проницаемости $\overline{\bar{\varepsilon}}$ (нижняя панель) от нормированных частот $\omega / \omega_{0 m}$ и $\omega / \omega_{c e}$ соответственно $\left(\Omega_{m u}=\omega_{m u} / \omega_{0 m}\right)$. Расчеты выполнены для $F=0.7, N=10^{17} \mathrm{~cm}^{-3}$ и $B_{0}=0.1 \mathrm{~T}$.

структуры вводится эффективная магнитная проницаемость среды, которая для идеально проводящих КРР определяется на основе следующего хорошо известного выражения [5]:

$$
\mu_{e f f}=1-\frac{F \omega^{2}}{\omega^{2}-\omega_{0 m}^{2}},
$$

где $F=\pi(r / T)^{2}, r$ - радиус внутреннего кольца, $T$ - период структуры, $\omega_{0 m}=c \sqrt{3 T /\left[\pi r^{3} \ln (2 w / \delta)\right]}-$ резонансная частота КРР, $w$ - ширина проводника, $\delta$ - радиальное расстояние между кольцами, $c-$ скорость света в свободном пространстве. Выражение (1) использовалось ранее в модели левой среды, созданной на основе изотропной плазмы $[5,6]$. Для того чтобы использовать выражение (1) в модели левой среды на основе анизотропной плазмы, необходимо создать условия, при которых диэлектрическая проницаемость анизотропной плазмы не сильно отличалась бы от изотропного случая. Такое условие может быть реализовано, если циклотронная частота электронов $\omega_{c e}$ намного меньше частоты $\omega_{0 m}\left(\omega_{c e} \ll \omega_{0 m}\right)$. При таком подходе предлагаемая модель может корректно описать только часть спектра ЭМВ, находящихся либо вблизи, либо выше частоты $\omega_{0 m}$.

Для расчета дисперсионных характеристик (ДХ) ЭМВ, существующих в продольно замагниченном плазменном метаматериале, воспользуемся дисперсионным уравнением для продольно замагниченной бесстолкновительной и холодной плазмы [9], которое с учетом эффективной магнитной проницаемости среды имеет следующий вид:

$$
\begin{gathered}
{\left[\eta k_{z}^{2}+\varepsilon\left(k_{x}^{2}-\mu_{e f f} k_{0}^{2} \eta\right)\right]\left(k_{z}^{2}+k_{x}^{2}-\mu_{e f f} k_{0}^{2} \varepsilon\right)} \\
+\mu_{e f f} k_{0}^{2} g^{2}\left(k_{x}^{2}-\mu_{e f f} k_{0}^{2} \eta\right)=0
\end{gathered}
$$

где $k_{x}$ и $k_{z}-$ поперечное и продольное волновые числа, $k_{0}=\omega / c-$ волновое число в вакууме, $\varepsilon=1+\omega_{p e}^{2} /\left(\omega_{c e}^{2}-\omega^{2}\right), g=-\omega_{p e}^{2} \omega_{c e} /\left[\left(\omega_{c e}^{2}-\omega^{2}\right) \omega\right]$, $\eta=1-\omega_{p e}^{2} / \omega^{2}-$ диагональные и недиагональные компоненты тензора $\overline{\bar{\varepsilon}}$, записанные для случая однокомпонентной (электронной) плазмы, $\omega_{p e}=\sqrt{4 \pi N e^{2} / m_{e}}-$ плазменная частота электронов, $N-$ концентрация электронов в плазме, $e / m_{e}-$ удельный заряд электрона, $\omega_{c e}=\left|e B_{0}\right| /\left(m_{e} c\right)$.

Будем рассматривать продольно замагниченный слой электронной плазмы, граничащий с обеих сторон с идеально проводящими металлическими экранами (полосковый волновод, полностью заполненный плазмой). При решении уравнений Максвелла в квазистатическом приближении $\left(k_{z} \gg k_{0}\right)$, которое является справедливым для тонких гиротропных слоев, функция поперечного волнового числа для полоскового волновода, полностью заполненного продольно намагниченной гиротропной 
$a$

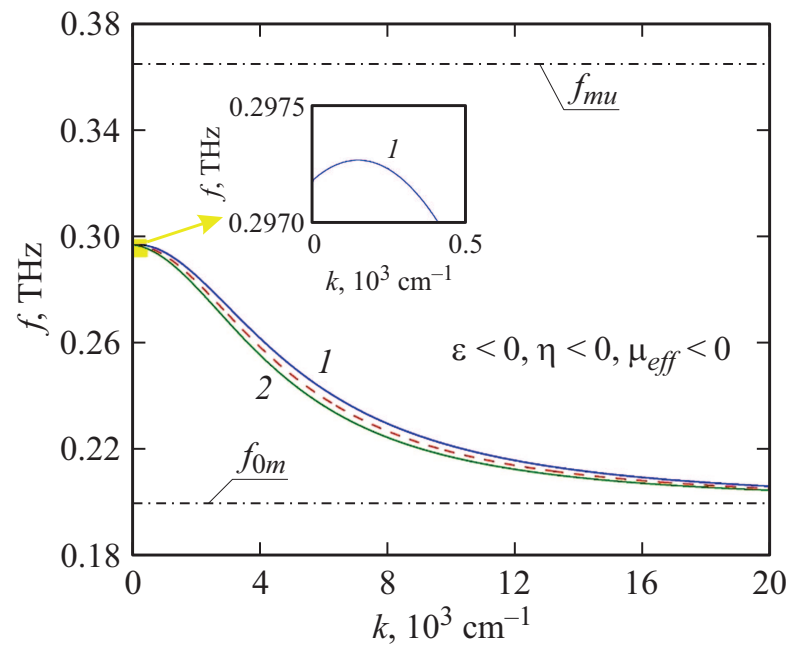

$b$

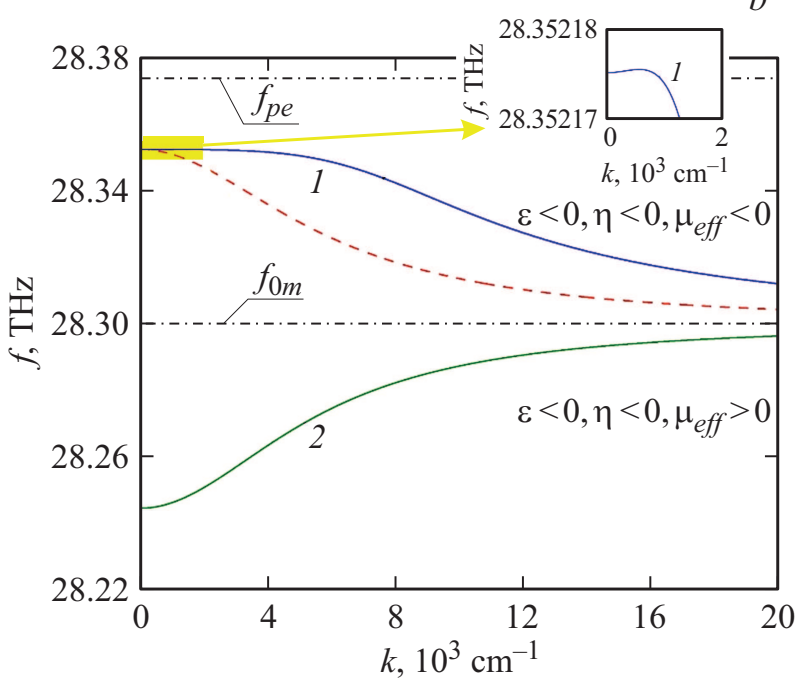

Рис. 2. Дисперсионные характеристики двух медленных объемных ЭМВ (1 - обыкновенной, 2 - необыкновенной), рассчитанные для левой среды на основе магнитоактивного плазменного метаматериала при двух значениях магнитной индукции и собственной частоты КРР: $a-B_{0}=1 \mathrm{~T}$ и $f_{0 m}=0.2 \mathrm{THz}, b-B_{0}=10 \mathrm{~T}$ и $f_{0 m}=28.3 \mathrm{THz}$. Расчеты на частях $a$ и $b$ выполнены для $d=10 \mu \mathrm{m}$, $n=1, N=10^{19} \mathrm{~cm}^{-3}$ и $F=0.7$. Штриховыми линиями показаны ДХ обыкновенной медленной объемной ЭМВ, существующей в левой среде на основе изотропного плазменного метаматериала.

средой, имеет вид

$$
k_{x}=\frac{n \pi}{d},
$$

где $d$ - толщина тонкого гиротропного слоя, $n-$ номер толщинной моды объемной ЭМВ. Для тонкого слоя магнитоактивной плазмы формула (3) справедлива для квази-ТМ ЭМВ.

Дисперсионное уравнение (2) с учетом (1) и (3) приводится к полиномиальному виду относительно круговой частоты $\omega$. Полином имеет шесть действительных положительных корней, два из которых соответствуют высокочастотным быстрым (обыкновенной и необыкновенной) объемным ЭМВ, фазовая скорость которых больше скорости света в свободном пространстве $\left(v_{p h}>c\right)$, а остальные четыре корня соответствуют медленным объемным ЭМВ, фазовая скорость которых значительно меньше скорости света в свободном пространстве $\left(v_{p h} \ll c\right)$. Две медленные ЭМВ являются хорошо известными плазменными волнами, одна из которых соответствует высокочастотной необыкновенной медленной ЭМВ, находящейся на частотах $\omega \sim \omega_{p e}$, а другая - низкочастотной медленной ЭМВ, находящейся на частотах $\omega<\omega_{c e}[10]$. Две оставшиеся медленные ЭМВ появляются за счет присутствия периодической структуры КРР внутри плазменного слоя.

На рис. $1, b$ приведены результаты расчета частотных зависимостей эффективной магнитной проницаемости и диагональной компоненты тензора $\overline{\bar{\varepsilon}}$ высокочастотной диэлектрической проницаемости. Из результатов расчета следует, что $\mu_{e f f}>0$ при $\omega<\omega_{0 m}$ и $\omega>\omega_{m и}$ и $\mu_{e f f}<0$ при $\omega_{0 m}<\omega<\omega_{m и}$, где $\omega_{m u}=\omega_{0 m} / \sqrt{1-F}$. Диагональная компонента $\varepsilon$ тензора $\overline{\bar{\varepsilon}}$ становится от- рицательной на частотах выше циклотронной частоты $\left(\omega>\omega_{c e}\right)$. Необходимо отметить, что вторая диагональная компонента $\eta$ тензора $\overline{\bar{\varepsilon}}$ будет меньше нуля $(\eta<0)$ при $\omega<\omega_{p e}$. Таким образом, для того чтобы реализовать из замагниченной электронной плазмы левую среду $\left(\varepsilon<0, \eta<0\right.$ и $\left.\mu_{e f f}<0\right)$, в которой будут распространяться медленные ЭМВ с отрицательной аномальной дисперсией, необходимо удовлетворить одному из двух следующих условий:

$$
\begin{aligned}
& \omega_{c e}<\omega_{0 m}<\omega<\omega_{m u}<\omega_{p e}, \\
& \omega_{c e}<\omega_{0 m}<\omega<\omega_{p e}<\omega_{m u} .
\end{aligned}
$$

На рис. $1, b$ заливкой выделена область частот, для которой условия (4a) и (4b) выполняются.

На рис. 2 приведены результаты расчета ДХ медленных ЭМВ, полученные на основе решения дисперсионного уравнения (2) с учетом (1) и (3). Расчеты выполнены для левой среды, созданной на основе как изотропного $\left(B_{0}=0\right)$, так и магнитоактивного $\left(B_{0} \neq 0\right)$ плазменного метаматериала. Сравнение показало, что в левой среде на основе изотропного плазменного метаматериала существует только одна (обыкновенная) медленная ЭМВ с отрицательной аномальной дисперсией. В случае левой среды на основе магнитоактивного плазменного метаматериала могут существовать как две (обыкновенная и необыкновенная) медленные ЭМВ (рис. 2,a), так и одна (обыкновенная) медленная ЭМВ (рис. 2,b). Первый случай реализуется при выполнении условия (4a), а второй - при выполнении условия (4b). Из представленных на вставках к рис. $2, a, b$ результатов также следует, что ДХ обыкновенной медленной 


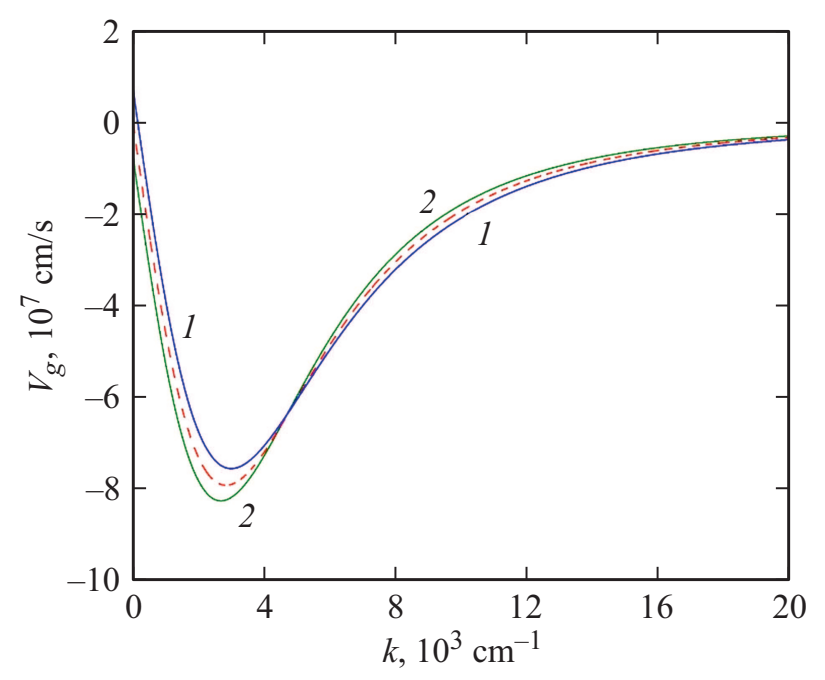

Рис. 3. Зависимости групповых скоростей $V_{g}$ от волнового числа $k$ двух медленных объемных ЭМВ $(1-$ обыкновенной, 2 - необыкновенной), существующих в левой среде на основе магнитоактивного плазменного метаматериала. Расчеты выполнены для $d=10 \mu \mathrm{m}, n=1, B_{0}=1 \mathrm{~T}, N=10^{19} \mathrm{~cm}^{-3}$, $f_{0 m}=0.2 \mathrm{THz}$ и $F=0.7$. Штриховой линией показана зависимость $V_{g}(k)$ обыкновенной медленной объемной ЭМВ, существующей в левой среде на основе изотропного плазменного метаматериала.

ЭМВ содержит участки как с отрицательной аномальной, так и с положительной нормальной дисперсией. Смена дисперсии наблюдается в относительно узкой области частот, появление которой не является следствием ошибки, возникающей в результате превышения машинной точности, так как последняя имеет величину 2.220446е-016. Напротив, необыкновенная медленная ЭМВ на частотах, где $\mu_{e f f}<0, \varepsilon<0$ и $\eta<0$, обладает только отрицательной аномальной дисперсией (рис. 2,a). Одновременное увеличение собственной частоты КРР и циклотронной частоты электронов приводит к тому, что необыкновенная медленная ЭМВ, смещаясь на частоты, где $\mu_{e f f}>0$, становится волной с положительной нормальной дисперсией (рис. 2,b). Как следует из результатов, представленных на рис. 3, максимальная величина групповой скорости необыкновенной медленной ЭМВ всегда больше, а обыкновенной медленной ЭМВ всегда меньше максимальной величины групповой скорости обыкновенной медленной ЭМВ, существующей в левой среде на основе изотропного плазменного метаматериала.

Необходимо отметить, что отличие диэлектрической проницаемости магнитоактивной плазмы от изотропной плазмы для двух рассмотренных выше случаев составляет величину не более $2 \%$ на собственной частоте КРР. Помимо этого существования медленных ЭМВ во всей полосе частот $f_{0 m}<f<f_{m и}$ можно достичь при $F \rightarrow 1$. Условие $T \ll \lambda$ ограничивает полосы частот медленных ЭМВ, в которых будут отсутствовать разрывы ДХ, обусловленные выполнением условий брэгговского резонанса для определенных значений волнового числа [11].

В заключение отметим, что проведенные в работе расчеты электродинамических характеристик медленных ЭМВ указывают на возможность использования магнитоактивной плазмы для создания управляемых магнитным полем левых сред в терагерцевом диапазоне частот. В качестве плазменных сред могут выступать не только газоразрядные среды [12], но и полупроводниковые материалы [13], для значения концентрации электронов которых и были проведены расчеты в данной работе.

\section{Финансирование работы}

Работа выполнена за счет гранта Российского научного фонда (проект № 19-79-20121).

\section{Конфликт интересов}

Авторы заявляют, что у них нет конфликта интересов.

\section{Список литературы}

[1] Tong X.C. Functional metamaterials and metadevices. Springer Ser. in Materials Science. Springer, 2018. V. 262. 277 p. https://doi.org/10.1007/978-3-319-66044-8

[2] Гуляев Ю.В., Лагарьков А.Н., Никитов С.А. // Вестник PAH. 2008. T. 78. № 5. C. 438-449. https://doi.org/10.1134/S1019331608030143

[3] Вендик И.Б., Вендик О.Г. // ЖТФ. 2013. Т. 83. В. 1. С. 3-28. https://doi.org/10.1134/S1063784213010234

[4] Веселаго В.Г. // УФН. 1967. Т. 92. № 7. С. 517-526. https://doi.org/10.3367/UFNr.0092.196707d.0517

[5] Pendry J.B., Holden A.J., Stewart W.J., Youngs I. // Phys. Rev. Lett. 1996. V. 76. N 25. P. 4773-4776. https://doi.org/10.1103/PhysRevLett.76.4773

[6] Smith D.R., Padilla W.J., Vier D.C., Nemat-Nasser S.C., Schultz S. // Phys. Rev. Lett. 2000. V. 84. N 18. P. 41844187. https://doi.org/10.1103/PhysRevLett.84.4184

[7] Амельченко М.Д., Гришин С.В., Шараевский Ю.П. // Письма в ЖТФ. 2019. Т. 45. В. 23. С. 14-18. https://doi.org/10.21883/PJTF.2019.23.48712.17830

[8] Ландау Л.Д., Лифбиии, Е.М. Теоретическая физика. Т. Х. Физическая кинетика. М.: Наука, 1979. 528 с.

[9] Еськин В.A. Электромагнитные волны в замагниченных плазменных волноводах. Н. Новгород: Нижегород. ун-т, 2012. $108 \mathrm{c}$.

[10] Электродинамика плазмы / Под ред. А.И. Ахиезера. 2-е изд. М.: Наука, 1974. 720 с.

[11] Kashyap R. Fiber Bragg gratings. San Diego: Academic Press, 1999. $458 \mathrm{p}$. https://doi.org/10.1016/B978-0-12-400560-0.X5000-3

[12] Sakai O., Tachibana K. // Plasma Sources Sci. Technol. 2012. V. 21. P. 013001. https://doi.org/10.1088/0963-0252/21/1/013001

[13] Киндяк А.С. // ЖТФ. 1994. Т. 64. В. 11. С. 99-104. 